\title{
Les vaincus du Golfe ou la guerre vue du Mozambique
}

Sergio Vieira

\section{OpenEdition}

1 Journals

\section{Édition électronique}

URL : http://journals.openedition.org/conflits/99

DOI : $10.4000 /$ conflits.99

ISSN : $1777-5345$

Éditeur :

CCLS - Centre d'études sur les conflits lilberté et sécurité, L'Harmattan

Édition imprimée

Date de publication : 17 mai 1991

ISSN : 1157-996X

\section{Référence électronique}

Sergio Vieira, "Les vaincus du Golfe ou la guerre vue du Mozambique », Cultures \& Conflits [En ligne], 02 I printemps 1991, mis en ligne le 30 décembre 2002, consulté le 30 mars 2021. URL : http:// journals.openedition.org/conflits/99; DOI : https://doi.org/10.4000/conflits.99

Ce document a été généré automatiquement le 30 mars 2021.

Creative Commons License 


\title{
Les vaincus du Golfe ou la guerre vue du Mozambique
}

\author{
Sergio Vieira
}

1 Selon certains, depuis un mois, la raison de la force essaie de s'imposer à la force de la raison. Une fois encore, la brutalité d'une science et d'une technologie militaire créerait les conditions d'une nouvelle humiliation des peuples arabes et imposerait un diktat additionnel aux peuples du tiers monde. D'autres affirment au contraire que le conflit n'oppose pas le Nord au Sud mais une coalition (Nord et Sud confondus) unie contre une grossière violation du droit des nations et que, de ce conflit, un nouvel ordre international plus pacifique pourra émerger. Sans trancher pour l'instant entre ces interprétations. En regardant simplement le film des événements précédents: l'invasion irakienne et le premier déluge de bombes sur Bagdad, on est contraint d'éprouver un certain malaise face aux occasions ratées de dialogue qui ont poussé l'ensemble des parties à choisir la seule alternative non raisonnable : la guerre. Avec en toile de fond, la position affichée par Bagdad de ne rien faire en faveur d'une diminution de la tension, la rencontre de Djeddah entre le vice-président Ezzat Ibrahim et le prince héritier (et Premier ministre) Saad, la veille de l'invasion irakienne au Koweït, succédant à l'étonnant dialogue du président Sadam Hussein avec Mme April Glaspie, l'ambassadeur américain à Bagdad, et à la position bienveillante du Département d'État par le biais des déclarations du sous-secrétaire John Kelly devant le sous comité du Moyen-Orient de la Chambre des représentants du Congrès des EtatsUnis, puis l'avortement du sommet organisé par le roi de Jordanie pour le 4 août, suite aux pressions exercées par Washington, enfin l'envoi de troupes avec une dimension offensive et le refus de laisser le temps nécessaire aux sanctions pour exercer leurs effets, puis pour terminer le changement continu des objectifs mêmes du conflit, sont autant d'éléments qui pointent en direction d'une volonté délibérée d'affrontement des Etats-Unis. D'ailleurs, le secrétaire général de l'ONU avouera au président irakien que l'assise internationale n'était pas en mesure de contrer la volonté américaine de mettre fin au régime. Mais l'Irak s'entêtera comme les Etats-Unis. Sûrs de leur technologie de pointe, ces derniers s'attendent à une guerre ultrarapide, gagnée dès les premières heures. Sûrs de l'incapacité politique de leurs adversaires de subir des pertes humaines, 
et convaincus que c'est l'occupation du terrain qui détermine en dernière analyse l'issue des conflits, les autres misent sur un système défensif bien testé. Une trentaine d'acteurs, plus ou moins actifs, s'affrontent dans le Golfe. Le reste des membres de la communauté internationale suit l'affaire, soit en première loge, puisque financièrement ils ont un mot à dire tels les Allemands et les Japonais ou parce que politiquement, on ne peut les ignorer : les Soviétiques et les Iraniens, soit de plus loin, parce qu'ils ne sont que des éléments de la périphérie sans emprise sur les événements. Vue de l'Afrique australe, vue du Mozambique, de la périphérie cette guerre ne peut être perçue que par les lourdes conséquences qu'elle entraîne sur des pays ayant besoin de l'attention internationale pour parachever des fragiles processus de paix et d'édification de sociétés pluralistes. En même temps, les coeurs serrés, on suit l'évolution des cours du pétrole et le rétrécissement de la capacité financière d'aide pour les victimes de la guerre et de la faim ainsi que pour la reconstruction et le développement.

Le manteau de la justice

2 L'évolution de la civilisation, du droit et de la conscience de l'homme ne tolère plus l'exercice brutal du massacre collectif qu'est la guerre. A la limite, on acceptera le sacrifice comme un moyen de mettre fin ou de prévenir une injustice ou une catastrophe encore plus importante. Ultima ratio, la guerre se voudra juste par ses buts et par ses moyens et conséquences, lorsqu'on sera dans l'impossibilité totale de trouver des solutions alternatives. Éprouvée par le colonialisme, convaincue que le partage du continent à Berlin est devenu un fait accompli, l'Afrique, dès 1963, lors de la création de l'OUA s'est refusée à mettre en cause les frontières héritées du colonialisme. Tout le monde est conscient qu'elles sont injustes, coupant de façon arbitraire des liens historiques et civilisationnels, voire même des relations familiales. Cependant, tous reconnaissent que l'alternative au statu quo serait des conflits sanglants, destructeurs et sans issue. La coopération régionale s'affirme alors comme la voie sage pour surmonter et ainsi rapprocher des familles, des ethnies, des cultures et des intérêts coupés. L'annexion d'une région, ou encore plus grave de tout un territoire, ou pire, d'un État, se heurte à un sentiment collectif de répulsion sur le continent.

3 Le Maroc (qui pourtant condamne l'annexion du Koweït et déplace 1200 hommes sur le Golfe), l'a appris à ses dépens lorsque l'Afrique refusa ses vues et appétits, jadis sur la Mauritanie et plus tard sur le Sahara occidental. Malgré le fait qu'à l'époque le Mozambique était une colonie portugaise, Nasser et Nyerere, lors du sommet de l'OUA au Caire en 1965, répondirent par une fin de non-recevoir aux visées expansionnistes du Président du Malawi sur le territoire. Par la suite, le Banda n'osera même pas soulever la question en public à ses pairs. Idi Amin avec l'action sur Kaguera en Tanzanie, Siad Barré lors de la tentative de s'emparer de l'Ogaden n'ont pas trouvé de soutien chez les instances de l'OUA. Dans le dernier cas cette position africaine est d'autant plus importante qu'en toile de fond, il y avait un sentiment africain hostile à la présence cubaine en Éthiopie et à la politique du bloc de l'Est et de l'URSS dans la Corne de l'Afrique.

4 Ainsi, et malgré les bonnes relations politiques, économiques que les uns et les autres entretenaient avec l'Irak, pas un seul État africain n'a soutenu ou légitimé l'action de Bagdad. On pourrait ajouter que tous les États de communauté internationale ont réagi de même. Nulle personnalité africaine ou mondiale n'a osé faire autrement. L'isolement politique et diplomatique, voir économique avec les sanctions, que connaît le régime du 
président Sadam Hussein, n'a pas d'égal dans l'histoire des nations. On ne saurait donc porter l'analyse ou la discussion sur la légitimité, ou la solidarité avec l'action irakienne. Elle est inadmissible, elle porte atteinte à plusieurs des principes essentiels $\mathrm{du}$ droit des Nations, des règles du jeu entre les États. En Afrique, elle est particulièrement ressentie, car elle viole le droit des peuples à l'autodétermination et à l'indépendance, elle met en cause l'intangibilité des frontières, elle s'arroge le droit de faire disparaître, par la force, un État, membre de l'ONU et ami du continent.

De ce point de vue, nul doute que l'action en cours est juste, surtout si l'on tient compte du fait que l'ONU, par le biais des résolutions du Conseil de Sécurité, a condamné l'Irak, exigé le retour au statu quo ante, imposé un embargo total, permis le recours à la force pour garantir sa mise en oeuvre et finalement autorisé l'usage de moyens militaires pour libérer le Koweït. Cependant, on décèle, de façon grandissante, voire majoritaire, dans l'opinion des pays de la périphérie non seulement un malaise face au bien-fondé de la guerre, mais aussi un sentiment d'admiration, ou même une certaine ferté à l'égard du défi irakien contre la super-puissance américaine. Cette réaction, à première vue illogique, s'enracine pourtant dans un raisonnement, plus consistant que les habituels clichés sur un anti-américanisme propagé par un "club des gueux et des fanatiques religieux" ne voudrait le laisser croire aux Etats-Unis.

On veut certes que l'action se destine à libérer le Koweït, puisqu'on ne peut tolérer l'annexion en totalité ou en partie d'un territoire. Et, en effet, nul n'accepte qu'après le colonialisme du Nord contre le Sud, lui succède un nouveau colonialisme et expansionnisme du Sud contre le Sud. Mais il irait alors de soi que cette règle, "sacrée", soit appliquée, en toute logique, dans toutes les circonstances identiques. Or, pendant des décennies, l'Afrique du Sud a conservé son annexion sur la Namibie, malgré maintes résolutions de l'ONU et ceci sans que grand mal lui advienne. Contrairement à l'Irak qui n'a porté la guerre que contre deux de ses voisins (l'Iran et le Koweït), Pretoria a commis des actes d'agression militaire contre tous les voisins, contre tous les États de la région australe, à l'exception de la Tanzanie et du Malawi (qui tout de même ont aussi subi les conséquences économiques de la stratégie globale), a soutenu des invasions mercenaires contre les Seychelles et Sao Tomé et Principe. La politique sudafricaine de déstabilisation militaire et économique a provoqué au Mozambique seul, un million de morts, cinq millions de déplacés ( $\mathrm{I} / 3$ de la population), plus de quinze milliards de dollars de destructions (plus de trois fois le total de la dette mozambicaine et plus de sept fois le $\mathrm{PNB}$ ), et cela sans grande réaction de la communauté internationale. En 1965, le gouvernement britannique a bien demandé au Conseil de sécurité d'appliquer des sanctions contre le régime minoritaire et raciste de Ian Smith en rébellion contre la couronne. Par la suite, le gouvernement de sa Majesté, pour garantir l'application de la résolution du Conseil de sécurité, a envoyé au large du port de Beira, au Mozambique, une frégate qui n'a jamais tiré un seul coup de feu. En 1976, lorsque le Mozambique a décrété les sanctions, le vaisseau fut retiré. (on ne saurait donc comparer ce que furent les sanctions contre la Rhodésie, voire l'Afrique du Sud et celles dont l'Irak fait l'objet.

7 Bien que, notamment le Mozambique et la Zambie aient été continuellement attaqués par les forces armées rhodésiennes, en dépit du fait que le Conseil de sécurité ait approuvé une résolution en faveur du renforcement de la capacité défensive du Mozambique, aucune solidarité ou soutien significatif de l'Occident ou même de la Grande-Bretagne n'eurent lieu. Les pertes subies par le Mozambique et la Zambie, en 
conséquence de l'application des sanctions et des actes rhodésiens de guerre, ne furent jamais objet d'une compensation autre que symbolique. A nouveau, on ne peut pas s'empêcher de comparer les situations. Toujours en Afrique, bien que refusée par l'OUA, la communauté des nations s'accommode de la présence marocaine au Sahara occidental.

En Asie, le gouvernement de l'Indonésie a militairement envahi et, par la force, annexé le Timor oriental en 1975, sous prétexte que les Portugais avaient abandonné le territoire. Jusqu'à aujourd'hui, on ne parvient même pas à inscrire l'affaire sur l'agenda de l'Assemblée générale de l'ONU. Plus récemment et déjà en pleine crise du Golfe, le gouvernement australien a ratifié, malgré la protestation portugaise, puissance administratrice, la signature d'un accord avec l'Indonésie sur le partage du pétrole de la plate-forme continentale du Timor oriental (Timor-Gap), au détriment des intérêts légitimes des mauberes. L'argument sous-jacent, c'est que le Timor oriental n'est qu'une colonie abandonnée par les Portugais. Ainsi, d'un trait de plume, le territoire et son peuple deviennent une res nullius, prête à être saisie en toute légalité par le premier passant. Au Moyen- Orient même, à l'orée de la zone du conflit, il semble que la légalité et la communauté internationale coexistent fort bien avec les agissements syriens au Liban, la présence turque à Chypre, et les annexions et occupations de territoires arabes par Israël, malgré des résolutions pertinentes de l'ONU et de son Conseil de sécurité. A part l'Islande il semble qu'aucune puissance ne se soit empressée de reconnaître l'indépendance des États baltes. D'autres violations du droit international, sur des principes aussi sensibles et vitaux, se sont déroulées devant l'impuissance de la communauté des nations.

On peut rappeler en passant les invasions de l'Afghanistan, de la Grenade, et du Panama (pour arrêter un chef d'État !), le minage des ports du Nicaragua, le soutien ouvert à des rébellions armées contre des gouvernements d'États membres de l'ONU. Chacun de ces actes viole la Charte, et porte atteinte à des principes essentiels. La réaction américaine peut, dans le cadre de cette logique, être perçue sous d'autres angles, que ceux du glaive de la justice et de la défense de la souveraineté des petits pays. I1 s'agirait d'abord d'affirmer une pax americana, lorsque le déclin soviétique conduit l'ancienne super-puissance à soumettre ses intérêts et alliances aux priorités américaines. De même, face à l'ascension financière, scientifique et technologique concurrentielles de l'Europe et du Japon, l'action armée viserait à consolider l'unipolarité américaine dans le domaine suprême de la force, où, jusqu'alors, seuls les Soviétiques se trouvaient en mesure de contester les États Unis.

Ce point de vue se trouve renforcé par le refus américain de patienter le temps suffisant pour que les sanctions puissent oeuvrer. On dirait que, dès les débuts de la crise, s'est affichée par les discours et les faits, la décision d'imposer le langage des armes. L'industrie d'armement qui envisageait avec détresse le déclin des commandes dans la période de "post-guerre froide", aurait poussée aussi vers des solutions radicales. L'obstination du président Sadam Hussein ne pourrait être présentée comme argument majeur, dès lors que les plus puissantes forces navales, aériennes et terrestres, imposaient le blocus et que la force de la réalité économique allait déterminer l'issue. Le but de la guerre peut être encore perçu comme une consolidation du contrôle des matières premières, en particulier le pétrole entre les mains des pays nantis du Nord, notamment les Etats-Unis. Le mot d'ordre des pacifistes d'Europe, "pas de sang contre du pétrole" pourrait aussi toucher le coeur du problème. Outre donc le 
contrôle des moyens financiers, scientifiques et technologiques, le Nord prétendrait alors renforcer son emprise sur les seules ressources du Sud, les ressources naturelles. Lorsque les murs et rideaux séparant l'Europe s'effondrent on pourrait, avec amertume, se dire qu'un nouveau mur et rideau bâti d'indifférence et de mépris s'érige entre le Nord et le Sud et que cette guerre est ainsi la première pierre du mur de l'ostracisme. L'évolution des buts de la guerre, de la libération du Koweït à l'anéantissement de la base économique et militaire de l'Irak, la création conséquente d'un nouveau facteur grave de déséquilibre régional que, seule la présence continuelle des Etats-Unis pourra limiter, laissent entendre que le nouvel ordre annoncé pourrait remettre sous tutelle un Sud affaibli.

11 A la brutalité militaire de l'Irak qui, par la force, met trait en cause des principes essentiels du droit des nations, correspondrait alors une action parallèle pour enlever au Sud les moyens de son développement, sa survie et sa dignité. On ne saurait justifier l'action par le besoin de stabiliser en faveur du Sud l'accès et les cours de cette matière première, dès lors que l'ensemble des prix des produits du Sud s'effondrent en termes réels. L'ampleur, dès les débuts de la crise de l'opposition à la guerre dans l'opinion du Nord, est indicatrice du malaise éthique et juridique qu'on éprouve, d'autant plus qu'elle est bien peu sympathique à la cause irakienne. Du pape au conseil des Églises, les voix des autorités morales ont été unanimes pour demander la substitution des combats par des négociations, même en préservant les moyens de pression. Ainsi et lorsqu'on est bien contraint de constater que deux poids et deux mesures oeuvrent sur la scène internationale, dans la périphérie, on s'interroge alors, pour savoir si respect du droit et des décisions du Conseil de sécurité, signifient seulement l'adhésion aux intérêts du Nord puissant. Force serait alors de dire que dans le conflit du Golfe, le noble manteau de la Justice se trouverait plutôt en haillons, quel qu'illégitime que soient les agissements irakiens.

La misère en haillons : les vrais perdants de la guerre du Golfe

Le Nord s'est empressé de soutenir financièrement les pays de la Ligne du front contre l'Irak, de compenser les pertes qu'ils subissent du fait des sanctions et même, dans le cas d'Israël, des destructions imposées à cet État, officiellement hors du conflit. Des milliards de dollars ont été avancés. Le Nord contribue financièrement, et autrement, à l'effort de guerre des forces alliées. En Afrique australe, on ne pourrait qu'être jaloux de cette solidarité si généreuse lorsque les intérêts des nantis sont en jeu et si maigre quand la dignité humaine niée par le racisme est en cause. Dans la région, où seul l'Angola est producteur de pétrole, la crise ne pouvait survenir à un pire moment. De par l'effet de la déstabilisation sud-africaine, qui s'est succédé à la confrontation rhodésienne et à la guerre coloniale portugaise, l'ensemble des pays se trouvent économiquement et socialement à bout de souffle. Trois décennies de violence ont dévasté la région. Les projections tanzaniennes indiquent que l'équivalent de $70 \%$ de la valeur totale des exportations de 1990 devraient être consacrées cette année à maintenir les niveaux d'importation de pétrole. L'inflation, qui serait passée de $30 \%$ en 1989 à $19,5 \%$ en 1990, pourrait d'après le ministère des Finances dépasser maintenant le cap des $30 \%$.

13 Si on ajoute que le service de la dette atteint déjà les $90 \%$ de la valeur des exportations, et si on essaie de mesurer les effets indirects de la hausse du prix de pétrole, combinés avec la réduction de l'aide extérieure, dont celle des Fonds du Koweït, on pourra saisir l'ampleur de l'effet de la crise. La Zambie recevrait son pétrole du Koweït à des prix 
subventionnés. Le ministre des Finances a rapporté au Parlement que, suite à la crise, l'addition du pétrole était passée de 100 millions de dollars en 1989 à 180 millions pour 1990 et prévoyait que la note de l'année en cours pourrait être doublée. Déjà, au niveau des prix aux consommateurs, les prix des produits raffinés étaient montés de plus de $300 \%$. Au Zimbabwe où les importations de pétrole représentaient $15 \%$ des importations, déjà on se propose des hausses de prix aux consommateurs des produits raffinés de l'ordre des $90 \%$. L'inflation qui se situait dans la case des $25 \%$ pourrait connaître des changements dramatiques. Avec la base industrielle la plus développée et l'agriculture la plus mécanisée, l'économie de Harare serait particulièrement vulnérable. La hausse des prix de l'énergie atteindraient sérieusement la performance des exportations. Au Mozambique, où les réserves de pétrole semblent être de moins d'une semaine, où quelque 75 à $80 \%$ des raffinés étaient importés de l'URSS dans le cadre d'un accord inter-gouvernemental à des conditions favorables, la situation est encore bien plus dramatique. Déjà, les prix au consommateur de l'essence dépassent un dollar par litre et celui du diesel atteint presque les 50 cents US. L'inf1ation qui, de 167 \% en 1987 était passée à $30 \%$ en 1989 et devait être ramenée à $18 \%$ en 1990, va à nouveau atteindre pour 90 le cap des $30 \%$. Le Premier ministre qui, à la fin du premier semestre, était optimiste sur la possibilité d'un taux de croissance de $5 \%$ pour 1990 , à la fin de l'année, estimait qu'on serait proche du $0 \%$ en termes réels. La situation de l'économie soviétique a déterminé une révision sérieuse des termes de la vente du pétrole au Mozambique.

Dans les conditions actuelles de l'économie Mozambicaine aux prix de 90, ce seraient la valeur totale des exportations qui devraient être affectées pour maintenir le même niveau d'importation de pétrole en termes pratiques. Des appels ont été adressés à la communauté internationale et certains pays, dont les pays nordiques et les Etats-Unis, se sont révélés sensibles à cet appel, mais il est douteux que ce soutien additionnel puisse compenser les pertes. La conjoncture affecte l'ensemble des mesures de recouvrement économique et social en cours en Afrique australe et il est possible d'affirmer que même les programmes de développement démocratique peuvent être touchés. Un exercice électoral, un effort de pluralisme en termes de société, le surgissement de plates-formes alternatives, sont plus que douteux lorsque les hommes vivent en dessous du seuil vital. Le Mozambique, qui en 30 années de guerres imposées a vu réduire le produit per capita de presque 250 dollars à 104 dollars, est presque dans l'impossibilité d'affronter les conséquences de cette guerre au Golfe.

Les effets du conflit du Golfe ne s'épuiseront donc pas avec le silence des armes. Au Golfe s'ajoutent d'autres priorités pour le Nord, à commencer par celle de diminuer les effets de la crise dans sa propre économie. Les intérêts stratégiques et économiques visant à consolider la nouvelle configuration de l'Est européen d'opérer la conversion économique de l'ancienne RDA intégrée dans la CEE, de maintenir les tendances positives en URSS, sont autant de facteurs qui marginalisent davantage l'intérêt qu'on peut accorder au Sud et à l'Afrique. La capacité d'aide et d'investissement du Nord se rétrécit. A eux seuls, les engagements allemands pour soutenir les alliés et les pays de la ligne du front du Golfe, dépassent la totalité du budget d'aide de la CEE pour l'ensemble des pays de l'ACP dans le cadre de Lomé IV Ainsi, quel que soit le vainqueur au Golfe, le vaincu c'est déjà la périphérie. Le spectre de la guerre s'est éveillé bien rapidement avec la fin de la confrontation bipolaire, et même si juridiquement elle ne met pas face à face le Nord et le Sud, il ne demeure pas moins que tous sont convaincus que sans la 
décision américaine elle n'aurait pas eu lieu et n'aurait pu en aucun cas prendre la présente ampleur de guerre mondiale, bien que sur un théâtre régional.

Vaincus, déçus, humiliés, regardant s'élever davantage le rideau entre le Nord et le Sud, se creuser de plus en plus le fossé entre riches et pauvres, grande devient l'attraction des idéologies fondamentalistes des idéologies extrémistes, des actions nihilistes. Avec l'attention de l'opinion concentrée sur le Golfe, le problème de la fin de l'apartheid passe au second plan dans les médias qui comptent. Ainsi diminuent les pressions contre le système. Sans que Mandela ait déjà acquis le droit de vote, on discute la fin des sanctions contre l'Afrique du Sud. Pour cela, on dira toujours qu'elles nuisent surtout aux Noirs et non aux élites au pouvoir, qu'après tout elles sont peu efficaces. Enfermés dans les maillons de la dette extérieure, soumis au contrôle d'un ordre économique mondial où ils ne jouent qu'un rôle mineur, les peuples du Sud et surtout de l'Afrique ont peu de raisons pour envisager l'avenir avec optimisme. Les recettes d'ajustement structurel de la Banque mondiale et du FMI, souvent contradictoires, sont impuissantes à apporter des contributions positives à une réalité africaine.

L'ensemble de l'Afrique sud-saharienne a un PNB égal à celui de la Belgique. Le scénario est sans nul doute bien pessimiste. D'autres voies existent cependant, si on est convaincu que nulle alternative à la paix et au dialogue n'est raisonnable. Le discours des armes doit être remplacé par celui des diplomates. Les sanctions et les destructions déjà existantes constituent autant de moyens de pression pour imposer le respect du principe inaliénable du droit des peuples à l'autodétermination et à l'indépendance, à des frontières reconnues et acceptées, à la sécurité. Mais ces droits doivent être reconnus à tous, y compris aux Palestiniens et aux Libanais. Les annexions sont intolérables aussi bien dans le Golfe qu'en Cisjordanie ou au Golan.

La persistance de l'injustice des deux poids, deux mesures, ne peut servir ni l'équilibre des intérêts ni l'apaisement des conflits. Ce sont des victoires à la Pyrrhus, celles qui se proposent de résoudre des conflits en en créant d'autres. Le droit des peuples à mettre leurs richesses naturelles au profit de leur essor économique et social ne peut pas être nié même lorsque les élites de ces mêmes peuples jouissent du statut de milliardaires du globe. Reste finalement le besoin de comprendre que seule notre planète peut être la maison commune de l'humanité et ainsi exiger la nécessaire solidarité, non seulement pour sauver les arbres, les baleines et les éléphants, mais surtout les hommes.

On se propose de construire un nouvel ordre international, cette guerre pourrait contribuer à y aboutir, à condition que le nouvel ordre ne devienne pas une tutelle imposée par les nantis aux pauvres, par une superpuissance aux peuples. Le nouvel ordre ne peut être trouvé que dans le respect du droit et de l'éthique, fondé sur l'équilibre des intérêts et la solidarité globale. Il exige qu'on emprunte les mêmes voies sur l'arène internationale que celles suivies par les nations pour installer et consolider l'État de droit, la Société civile. La primauté de la loi, l'égalité devant elle, la tolérance de la différence, le pluralisme et l'exercice du pouvoir de police par les États et non par les individus ou groupes et clans, sont autant d'éléments à introduire, lorsqu'on veut réfléchir sur la configuration à apporter aux relations internationales. Les pays de la périphérie comptent bien peu aux centres de décision de la planète, pourtant la majorité écrasante de l'humanité y habite, et s'y trouvent des ressources humaines, des ressources de cerveau nécessaires à l'essor de l'Homme. Le nouvel ordre pour être stable et, source d'équilibre doit se construire sur cette égalité et cette solidarité des nations et des hommes. 


\section{RÉSUMÉS}

Vue d'Afrique australe, particulièrement du Mozambique, la Guerre du Golfe ne peut être perçue qu'à travers ses lourdes conséquences sur les états africains nécessitant l'aide internationale pour mener à bien de fragiles processus de paix. Elles sont, en effet, épouvantables, tant sur les plans financier que politique. Le combat contre l'Apartheid est relégué à l'arrière-plan de la scène internationale. Politiquement et économiquement oubliés, le Mozambique et tous les états voisins sont les grands perdants de la guerre. Si personne n'approuve vraiment Saddam Hussein, dans une partie de l'Afrique où la grande puissance locale, l'Afrique du Sud, n'a jamais cessé de violer les frontières avec ses voisins, le soutien local à l'Irak plonge ses racines dans le refus des pays pauvres d'être sous la tutelle des pays riches.

Viewed from Austral Africa, particularly Mozambique, the Gulf War cannot be appraised solely by its heavy consequences on African states needing international care to achieve fragile peace processes. Either on financial or on political levels, consequences are tremendous. The fight against apartheid has been dismissed to the backstage of the international scene. Politically and economically forgotten, Mozambique, as all the other peripheral States, are the great losers of the war. If nobody really approves of Saddam Hussein in a part of Africa where the leading regional power, South Africa, has never stopped transgressing its neighbour's borders, local support for Iraq deepens its roots in the refusal of a tutelage by the Rich of the Poor.

\section{INDEX}

Index géographique : Afrique australe, Mozambique

Mots-clés : conflits internationaux, guerre

Index chronologique : 1991

\section{AUTEUR}

\section{SERGIO VIEIRA}

Directeur du Centre d'Etudes africaines de l'Université d'Eduardo Mondlane au Mozambique 